\title{
Falsely Elevated Creatinine on Enzymatic Assay in a Patient Receiving Subcutaneous IgG Therapy
}

\author{
Ashley R. Santilli, MD ${ }^{7}$ (D) and Mark L. Wieland, $M D^{2}$ \\ 'Department of Neurology, Mayo Clinic, 200 1st St. SW, Rochester, MN, USA; ²Department of Internal Medicine, Mayo Clinic, Rochester, MN, USA.
}

J Gen Intern Med 36(5):1427-8

DOI: $10.1007 / \mathrm{s} 11606-021-06631-\mathrm{w}$

(c) Society of General Internal Medicine 2021

\section{INTRODUCTION}

Acute kidney injury (AKI) is characterized by a rapid decline in renal function measured by decreased glomerular filtrate rate (GFR), increased serum creatinine, increased serum blood urea nitrogen (BUN), and decreased urine output . ${ }^{1}$ Work up to uncover the etiology of AKI can involve evaluation of recent clinical history, past medical history, medication review, physical exam, serum and urine studies, imaging including renal ultrasound, and renal biopsy ${ }^{2}$ If there are concerns that the enzymatic serum creatinine assessment may be inaccurate, other diagnostics are performed. Cystatin $\mathrm{C}$ levels are determined almost entirely by glomerular filtration and are not affected by the influence of diet, body muscle mass, and gender, unlike serum creatinine.$^{3}$ Additionally, cystatin $\mathrm{C}$ and creatinine by Jaffe are not assessed via enzymatic assays like serum creatinine which may be susceptible to interactions based on patient factors or current treatments . ${ }^{4-6}$

\section{CASE DESCRIPTION}

A 78-year-old woman was directly admitted to the hospital from clinic for incidentally discovered asymptomatic stage III non-oliguric AKI with creatinine $3.93 \mathrm{mg} / \mathrm{dL}$, increased from $0.86 \mathrm{mg} / \mathrm{dL}$ (assessed at an outside institution) approximately 1 month prior. She denied any associated symptoms and was in her usual state of health. Her medical history was significant for chronic inflammatory demyelinating polyneuropathy for which she was on treatment with subcutaneous IgG (Hizentra® 14 g weekly).

Extensive workup was unrevealing as documented in Table 1. Prior to pursuing renal biopsy, decision was made to assess cystatin $\mathrm{C}$ and creatinine by Jaffe given the prior case report of IgG therapy interacting with enzymatic creatinine assays. A single blood sample result showed cystatin C $1.13 \mathrm{mg} / \mathrm{L}$ (eGFR 57) and enzymatic creatinine $3.7 \mathrm{mg} / \mathrm{dL}(\mathrm{eGFR}<15)$. A subsequent single

Received May 26, 2020

Accepted January 17, 2021

Published online February 5, 2021 blood sample showed creatinine by Jaffe $0.7 \mathrm{mg} / \mathrm{dL}$ and enzymatic creatinine assay $3.32 \mathrm{mg} / \mathrm{dL}$. Therefore, we concluded that the patient's elevated enzymatic creatinine was a false elevation and she was discharged to home.

Upon chart review, in 2017 the same patient was receiving IVIG therapy. She had an enzymatic creatinine of $2.0 \mathrm{mg} / \mathrm{dL}$ and 2 days later was found to have creatinine by Jaffe of 0.5 $\mathrm{mg} / \mathrm{dL}$.

\section{DISCUSSION}

To our knowledge, this case represents the second patient reported in the literature with falsely elevated enzymatic creatinine while receiving IgG therapy . ${ }^{4}$ Our patient demonstrated these phenomena with both intravenous and subcutaneous IgG preparations, in 2017 and 2019 respectively.

The enzymatic creatinine assay utilized at our institution is measured using the Roche P-Modular analyzer (Roche Diagnostics) . ${ }^{7}$ According to their package insert, the assay is assessed by measuring the conversion of creatinine with the aid of creatininase, creatinase, and sarcosine oxidase to glycine, formaldehyde, and hydrogen peroxide. The liberated hydrogen peroxide reacts with 4-aminophenazone and hydroxyl(tosyloxy)iodobenzene (HTIB) to form a quinone

Table 1 Summary of Extensive Evaluations Completed for Workup of Acute Renal Failure

\begin{tabular}{ll}
\hline \hline Test & Result (reference range and units) \\
\hline Urinalysis & Unremarkable \\
24 h urine creatinine & $1071(601-1689 \mathrm{mg} / 24 \mathrm{~h})$ \\
24 h urine protein & $190(<229 \mathrm{mg} / 24 \mathrm{~h})$ \\
Creatinine kinase & $62(26-192 \mathrm{U} / \mathrm{L})$ \\
Total complement & $57(30-75 \mathrm{U} / \mathrm{mL})$ \\
C3 & $84(75-175 \mathrm{mg} / \mathrm{dL})$ \\
C4 & $12(14-40 \mathrm{mg} / \mathrm{dL})$ \\
Cryoglobulin & Negative \\
Total protein & $7.2(6.3-7.9 \mathrm{~g} / \mathrm{dL})$ \\
ADAMTS13 activity & $>100(>70 \%)$ \\
Antinuclear antibody & $0.7(<1.0 \mathrm{U})$ \\
Rheumatoid factor & $<15(<15 \mathrm{IU} / \mathrm{mL})$ \\
Anti-cyclic citrullinated & $<15.6(<20.0 \mathrm{U})$ \\
peptide & $4.8(4.0-5.6 \%)$ \\
Hemoglobin A1c & $2.3(0.3-4.2 \mathrm{mlU} / \mathrm{L})$ \\
Thyroid stimulating & \\
hormone & $1.84(0.33-1.94 \mathrm{mg} / \mathrm{dL})$ \\
Kappa free light chains & $1.04(0.57-2.63 \mathrm{mg} / \mathrm{dL})$ \\
Lambda free light chains & No evidence for medical renal disease or \\
Renal ultrasound & vascular compromise \\
&
\end{tabular}


imine chromogen. The color intensity of the quinone imine chromogen is directly proportional to the creatinine concentration in the reaction mixture ${ }^{8}$ Proline is a low efficiency substrate for sarcosine oxidase; therefore, proline may be processed by sarcosine oxidase, leading to an additional source of hydrogen peroxide, which results in increased production of chromogen, thus falsely increasing the creatinine measurement.$^{4}$ Proline concentrations above $1 \mathrm{mmol} / \mathrm{L}$ are listed as positive interfering substances on the Roche Diagnostics package insert. ${ }^{7}$ Creatinine by Jaffe and cystatin $\mathrm{C}$ are not susceptible to this false increase in creatinine as they are not enzymatic assays.${ }^{5,6}$ Both Privigen ${ }^{\circledR}$ (IVIG) and Hizentra ${ }^{\circledR}$ (subcutaneous $\mathrm{IgG}$ ) contain approximately 250 $\mathrm{mmol} / \mathrm{L}$ of L-proline as a stabilizer ${ }^{9,10}$ The half-life of the interfering proline in these $\operatorname{IgG}$ preparations is not currently known and therefore, the ideal timing for assessing creatinine via the enzymatic assay in patients receiving these treatments is not clear.

The creatinine by the Jaffe method had been the cornerstone of creatinine measurement for many years.${ }^{11}$ Described by Max Jaffe, a pharmacologist, biochemist, and pathologist in 1886 , the method involves creatinine reacting with picric acid to produce a colored complex that can be quantified.$^{12}$ Despite many changes to the assay, there continue to be many drugs and disease states that are known to interact with the creatinine by the Jaffe assay. Examples of interfering medications include cefazolin and barbiturates and interfering disease states include diabetic ketoacidosis and hemolysis.$^{13}$ Picric acid can react with non-creatinine chromogens including ketoacids, fasting, lipemia, and hemolysis.$^{12,13}$ Additionally, picric acid can react with other substances, like cephalosporin antibiotics, to form non-creatinine chromogens.$^{14}$ Therefore, new assays, including the enzymatic assay described above, were created to more specifically measure creatinine concentration without the unwanted interference described in the Jaffe method.

The inability to accurately measure creatinine by the enzymatic method is not limited to increased proline in the blood. Saenger et al. (2009) described negative interferences in patients receiving catecholamines, most notably with dopamine and dobutamine, such that their creatinine measurements by the enzymatic method were significantly lower than the creatinine by Jaffe methods.$^{15}$ The mechanisms for negative interference of these catecholamines are distinct; Dobutamine is rapidly oxidized by hydrogen peroxide resulting in decreased hydrogen peroxide necessary to generate the measured chromogen and dopamine reacts with certain substrates in the presence of hydrogen peroxide to form a novel quinone imine dye with smaller absorptivity resulting in reduced total absorbance at the wavelength used to measure the chromogen.$^{16}$ Health care providers should be aware of the interference caused by proline and catecholamines in the enzymatic creatinine assay and assess creatinine using other methods to avoid potentially over- or under-diagnosing renal dysfunction.

\section{CONCLUSION}

This is the second case reported in the literature that identified falsely elevated enzymatic creatinine in a patient receiving IgG therapy. Both subcutaneous and intravenous forms of $\operatorname{IgG}$ contain the stabilizer L-proline which can cause increased proline in the blood and interact with enzymatic creatinine assays, leading to falsely elevated values. Therefore, providers caring for patients with AKI of unclear etiology receiving IgG therapy should consider assessing their renal function with non-enzymatic assays, like creatinine by Jaffe or cystatin C, to prevent unnecessary testing and treatment.

Corresponding Author: Ashley R. Santilli, MD; Department of Neurology, Mayo Clinic, 200 1st St. SW, Rochester, MN, USA (e-mail: Santilli.Ashley@mayo.edu).

\section{Declarations:}

Conflict of Interest: The authors declare that they do not have a conflict of interest.

\section{REFERENCES}

1. Farrar A. Acute kidney injury. Nursing clinics of North America. 2018. 53(4):499-510.

2. Koza, Y. Acute kidney injury: current concepts and new insights. J Inj Violence res. 2016. 1:58-62.

3. Teo SH, Endre ZH. Biomarkers in acute kidney injury (AKI). Best Pract Res Clin Anaesthesiol. 2017. 31(3):331-334.

4. Knezevic CE, Ness MA, Kratz LE, Marzinke MA. Elevated creatinine in a patient on IVIG-therapy. Clin Chim Acta. 2018. 486:94-97.

5. Cystatin C with Estimated GFR, Serum. Mayo Clinic Laboratories. 2011. Gentian cystatin C immunoassay on Cobas c501, Roche Diagnostics. Mayo Clinic Lab Test Catalog

6. Creatinine, Jaffe, Plasma. Mayo Clinic Laboratories. 2016. Package insert: Creatinine Jaffe Gen. 2 Method Sheet, Roche Diagnostics. Mayo Clinic Lab Test Catalog

7. Creatinine. Test principle: enzymatic colorimetric. Roche Diagnostics. GmbH. 2011. Available at: https://custombiotech.roche.com/content/ dam/internet/dia/custombiotech/custombiotech_com/en_GB/pdf/ CustomBiochech_Creatinine_TestFlyer.pdf. Accessed May 5, 2020.

8. Basic Metabolic Panel, Serum. Mayo Clinic Laboratories. 2016: Mayo Clinic Lab Test Catalog

9. Hizentra ${ }^{\circledR}$ Immune Globulin Subcutaneous (Human) (IGSC), 20\% Liquid. Full Prescribing Information. CSL Behring. Revised 2015. Available at: https://www.privigen.com/common/pdf/privigen-manufacturing-proline-brochure.pdf. Accessed November 28, 2020.

10. Privigen ${ }^{\circ}$, Immune Globulin Intravenous (Human), 10\% Liquid. Full Prescribing Information. CSL Behring. Revised 2013. Available at: https://www.privigen.com/common/pdf/privigen-manufacturing-proline-brochure.pdf. Accessed November 28, 2020.

11. Peake M, Whiting $\mathbf{M}$. Measurement of serum creatinine-current status and future goals. Clin Biochem Rev. 2006. 27(4):173-84.

12. Delanaye $\mathbf{P}$, Cavalier E, Pottel H. Serum creatinine: not so simple! Nephron. 2017. 136:302-308.

13. Samra M, Abcar AC. False estimates of elevated creatinine. Perm J. 2012. 16(2):51-2.

14. Syal K, Banerjee D, Srinivasan A. Creatinine estimation and interference. Indian Journal of clinical Biochemistry. 2013. 28(2):210-211.

15. Saenger AK, Lockwood C, Snozek CL, et al. Catecholamine interference in enzymatic creatinine assays. Clin Chem. 2009. 55(9):1732-6.

16. Karon BS, Daly TM, Scott MG. Mechanisms of dopamine and dobutamine interference in biochemical tests that use peroxide and peroxidase to generate chromophore. General Clinical Chemistry. 1998. 44(1):155-160.

Publisher's Note: Springer Nature remains neutral with regard to jurisdictional claims in published maps and institutional affiliations. 